\title{
ARTICLE OPEN \\ The vicK gene of Streptococcus mutans mediates its cariogenicity via exopolysaccharides metabolism
}

\author{
Yalan Deng ${ }^{1}$, Yingming Yang ${ }^{1}$, Bin Zhang ${ }^{1}$, Hong Chen ${ }^{1}$, Yangyu Lu ${ }^{1}$, Shirui Ren ${ }^{1}$, Lei Lei ${ }^{1 凶}$ and Tao Hu ${ }^{1 凶}$
}

\begin{abstract}
Streptococcus mutans (S. mutans) is generally regarded as a major contributor to dental caries because of its ability to synthesize extracellular polysaccharides (EPS) that aid in the formation of plaque biofilm. The VicRKX system of $S$. mutans plays an important role in biofilm formation. The aim of this study was to investigate the effects of vicK gene on specific characteristics of EPS in S. mutans biofilm. We constructed single-species biofilms formed by different mutants of vick gene. Production and distribution of EPS were detected through atomic force microscopy, scanning electron microscopy and confocal laser scanning microscopy. Microcosmic structures of EPS were analyzed by gel permeation chromatography and gas chromatography-mass spectrometry. Cariogenicity of the vicK mutant was assessed in a specific pathogen-free rat model. Transcriptional levels of cariogenicityassociated genes were confirmed by quantitative real-time polymerase chain reaction. The results showed that deletion of vicK gene suppressed biofilm formation as well as EPS production, and EPS were synthesized mostly around the cells. Molecular weight and monosaccharide components underwent evident alterations. Biofilms formed in vivo were sparse and contributed a decreased degree of caries. Moreover, expressional levels of genes related to EPS synthesis were down-regulated, except for gtfB. Our report demonstrates that vick gene enhances biofilm formation and subsequent caries development. And this may due to its regulations on EPS metabolism, like synthesis or microcosmic features of EPS. This study suggests that vick gene and EPS can be considered as promising targets to modulate dental caries.
\end{abstract}

International Journal of Oral Science (2021)13:45

https://doi.org/10.1038/s41368-021-00149-x

\section{INTRODUCTION}

Dental caries is a chronic infectious disease occurring on mineralized tooth tissue, which is considered one of the most common health problems globally in 2015 posing considerable economic, social, and health-related burden. ${ }^{1-3}$ Streptococcus mutans ( $S$. mutans) is important in caries development because of its ability to synthesize extracellular polysaccharides (EPS) matrix. ${ }^{4}$ EPS, with all the oral microorganisms embedding in ${ }^{5}$ is essential in oral microenvironment, because it can form a well-structured biofilm on the tooth surface. ${ }^{1,6,7}$ It can also function as a sensor for environmental signals and provide a long-term dynamic protection for microbial communities. ${ }^{6,8}$ Nowadays, with the thriving development of targeted therapies, regulations on EPS metabolism and biofilm formation have gained considerable attention. ${ }^{9,10}$ Nanoparticles have been used to disrupt biofilm and a small molecule which can selectively target $S$. mutans biofilms has also been reported to effectively reduce dental caries in vivo without affecting the overall oral microbiota. ${ }^{11,12}$ Researchers have found that the production, distribution as well as microcosmic characteristics of EPS all take a considerable part in the pathogenicity of polymicrobial biofilm. . $^{13,14}$ So, an understanding of specific characteristics of EPS derived from S. mutans biofilm will provide more solid evidences for caries prevention, which have not been elucidated yet.

VicRKX signal transduction system consists of three encoding proteins: VicK, a histidine protein kinase; VicR, a global response regulator (RR); and VicX, a putative hydrolase. ${ }^{15-17}$ VicK, as an essential link between the environmental stimuli and cellular status, is supposed to sense and transmit chemical signals to downstream regulatory proteins, like VicR and GcrR. ${ }^{18,19}$ And subsequently, it can regulate transcriptional levels of biofilm associated genes, like $g t f B / C$, $f t f$ and $g b p B .^{20-22}$ As a result, vick gene possesses an important position in multiple bioactivities of S. mutans, particularly biofilm formation. ${ }^{23,24}$ What's more, an epidemiological research has reported that the frequency of a vick C470T missense mutation was higher in the high-severity caries group than in the caries-free group. ${ }^{25}$ The mutation of C470T contributes to the alternations of PAS domain of VicK protein, and PAS domain is a major sensor to environmental stresses, ${ }^{26}$ which means vick gene, as well as VicK protein are associated with the cariogenicity of $S$. mutans closely.

At present, few studies on the relationship between microcosmic structures of EPS and cariogenicity of $S$. mutans have been found. Although vick gene can modulate biofilm formation and virulence of $S$. mutans in some degree, it is not clear whether this modulation is related to EPS metabolism, especially its structural characteristics. And the comprehensive regulation among vick gene, transcriptional levels of downstream genes, EPS synthesis and cariogenicity of $S$. mutans remains unknown. Hence, this study aimed to identify the role of vick gene in the cariogenicity of S. mutans in vitro and in vivo, as well as to elucidate effects of vicK gene on the EPS.

\footnotetext{
${ }^{1}$ State Key Laboratory of Oral Diseases \& National Clinical Research Center for Oral Diseases \& Department of Preventive Dentistry, West China Hospital of Stomatology, Sichuan University, 610041 Chengdu, China

Correspondence: Lei Lei (leilei@scu.edu.cn) or Tao Hu (hutao@scu.edu.cn)

These authors contributed equally: Yalan Deng, Yingming Yang
}

Received: 8 December 2020 Revised: 1 July 2021 Accepted: 25 October 2021

Published online: 16 December 2021 
a

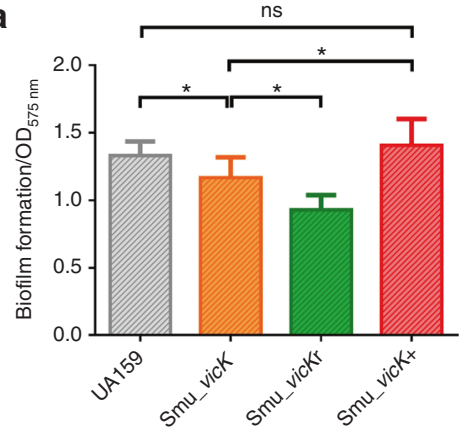

C

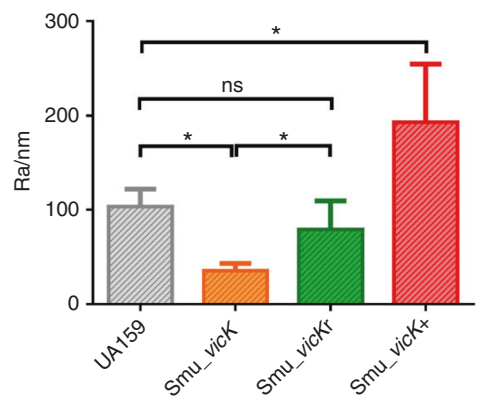

d

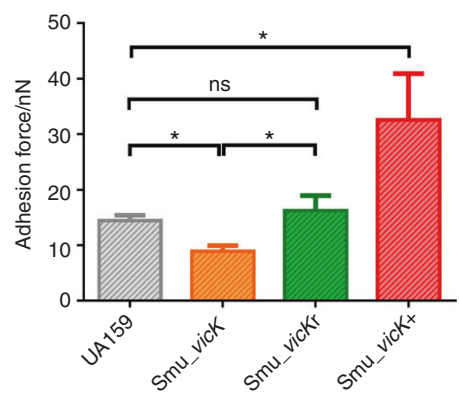

b
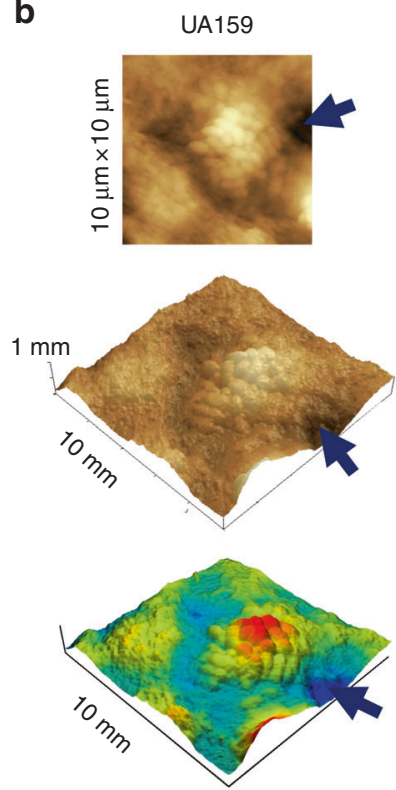

Smu_vicK

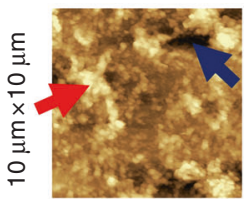

Smu_vicKr

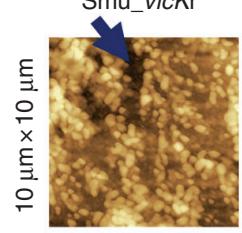

Smu_vicK+
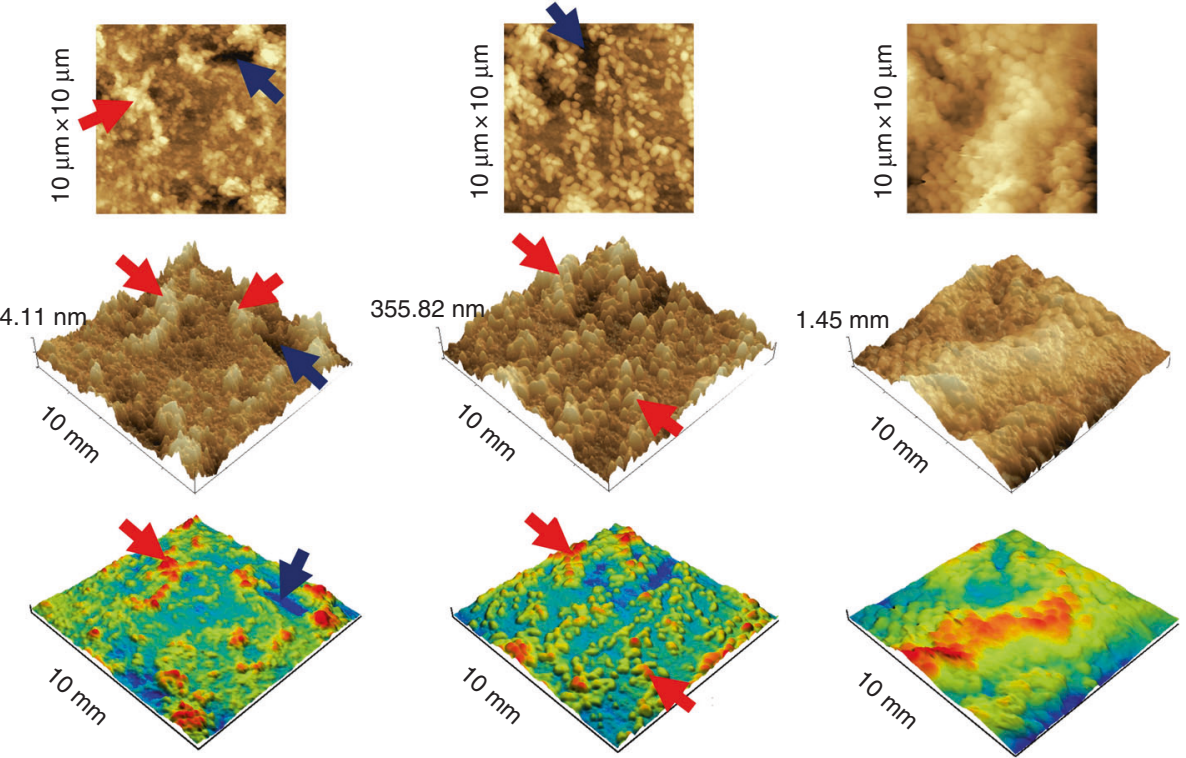

Fig. 1 Analysis of biofilm morphologies. Unpaired $t$ test was used to detect the statistical significances. a Biomass was quantified by CV staining, and the optical density at $575 \mathrm{~nm}$ was read. $\left(n=6 ;{ }^{*} P<0.05\right.$; ns no significant difference). b Surface characteristics and the $3-\mathrm{D}$ reconstruction of $24 \mathrm{~h}$ biofilms (an area of $10 \mu \mathrm{m} \times 10 \mu \mathrm{m}$, with a bar indicating vertical deviations) with the light (red) and dark (blue) zone representing peaks and valleys. c Surface roughness (Ra) of biofilms was obtained via AFM. ( $n=4$; ${ }^{*} P<0.05$; ns no significant difference). d Adhesion force data were obtained from AFM. $\left(n=3 ;{ }^{*} P<0.05\right.$; ns no significant difference)

\section{RESULTS}

vick was involved in biofilm morphological changes

To compare the abilities of different strains to form biofilms, crystal violet (CV) assay was used and the results showed that the mean value of Smu_vicK (the vicK deficient strain) biofilms was statistically lower than that of UA159 (Fig. 1a). Atomic force microscopy (AFM) observations indicated a peak-and-valley topography in Smu_vicK and Smu_vicKr (the vicK complementary strain) biofilms (peaks: red arrows; valleys: dark blue arrows; Fig. 1b). UA159, as well as the Smu_vicK+ (the vick overexpression strain) biofilms had smoother and more flourishing surface (Fig. 1b). Mean Ra of Smu_vick biofilms was markedly lower than the UA159 group, while that of Smu_vicK+ was significantly higher and that of Smu_vicKr was not statistically different (Fig. 1c). Adhesion force of Smu_vicK biofilms was statistically lower, whereas Smu_vicK+ appeared an enhanced capacity (Fig. 1d).

vicK changed production and distribution of EPS in biofilm Compared with UA159, Smu_vicK biofilms demonstrated an aberrant architecture with more scattered microcolonies and thinner EPS (Fig. 2a). Evidently, Smu_vicK biofilms seemed to be devoid of EPS when scanned under a higher magnification $(5000 \times)$. On the other hand, Smu_vicK+ cells were densely encased by enriched EPS (Fig. 2a). Confocal laser scanning microscopy (CLSM) data showed that only small clusters of bacterial cells were observed in Smu_vicK and Smu_vicKr biofilms and EPS were synthesized mostly around the cells, while there were much more EPS enmeshing the bacterial aggregates and filling the space between them in UA159 and Smu_vicK+ biofilms (Fig. 2b). As shown in Fig. 2c, both EPS and bacterial cells of Smu_vick and Smu_vicKr biofilms were less than those of UA159 and Smu_vicK+ biofilms. EPS/bacteria ratios of the biofilm formed by Smu_vick were significantly lower than those of UA159 and Smu_vicKr.

Analyses of microcosmic structures of EPS generated from biofilms $\mathrm{MW}_{\mathrm{w}}$ (weight-average molecular weight) and $\mathrm{MW}_{\mathrm{n}}$ (number-average molecular weight) were demonstrated in Fig. 3. Water-soluble glucans (WSGs) and water-insoluble glucans (WIGs) isolated from Smu_vick biofilms presented the highest $\mathrm{MW}_{\mathrm{w}}$ (5 942.2 Da and 5 938.2 Da, respectively), while those from Smu_vicK+ illustrated the lowest MW $_{\mathrm{w}}$ when UA159 was as a reference (Fig. 3a, b). For further analysis, the mean molecular weight of WSGs from Smu_vicK biofilms were statistically higher than those from UA159 biofilms (Fig. 3c).

Mannose and glucose were main constituents in both WSGs and WIGs (Fig. 4). Particularly, Smu_vicK WSGs consisted of lower mannose $(15.55 \% \pm 0.36 \%)$, higher glucose $(84.46 \% \pm 0.36 \%)$ and none galactose when compared to UA159 WSGs, in which 
a
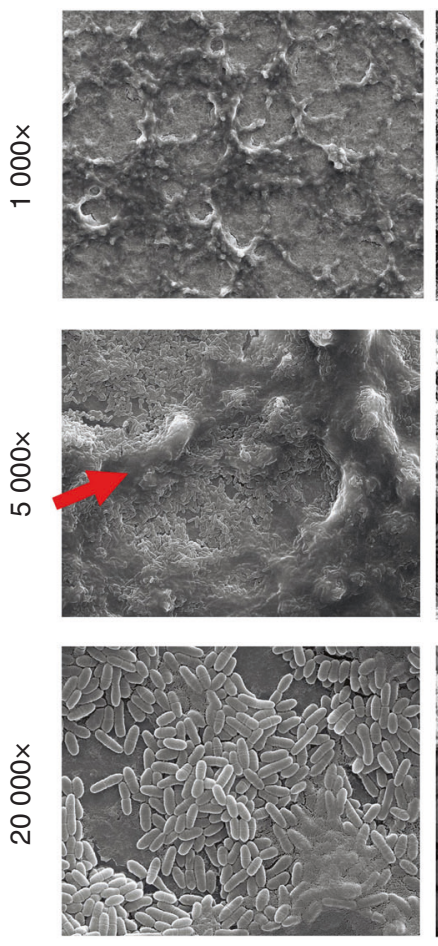

Smu_vicKr
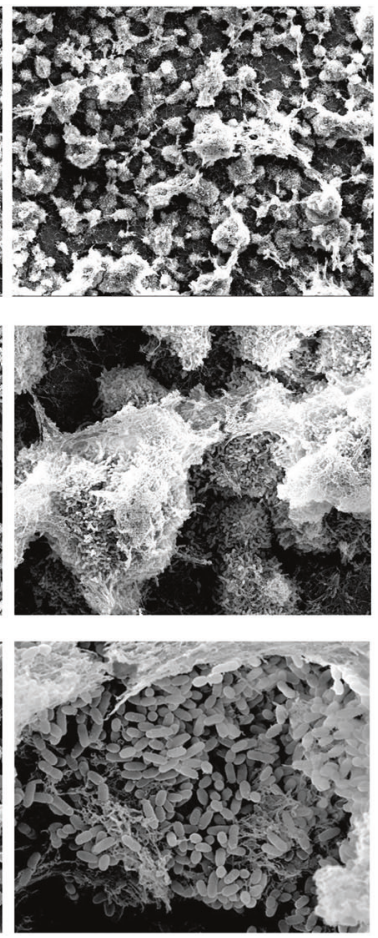

Smu_vicK+
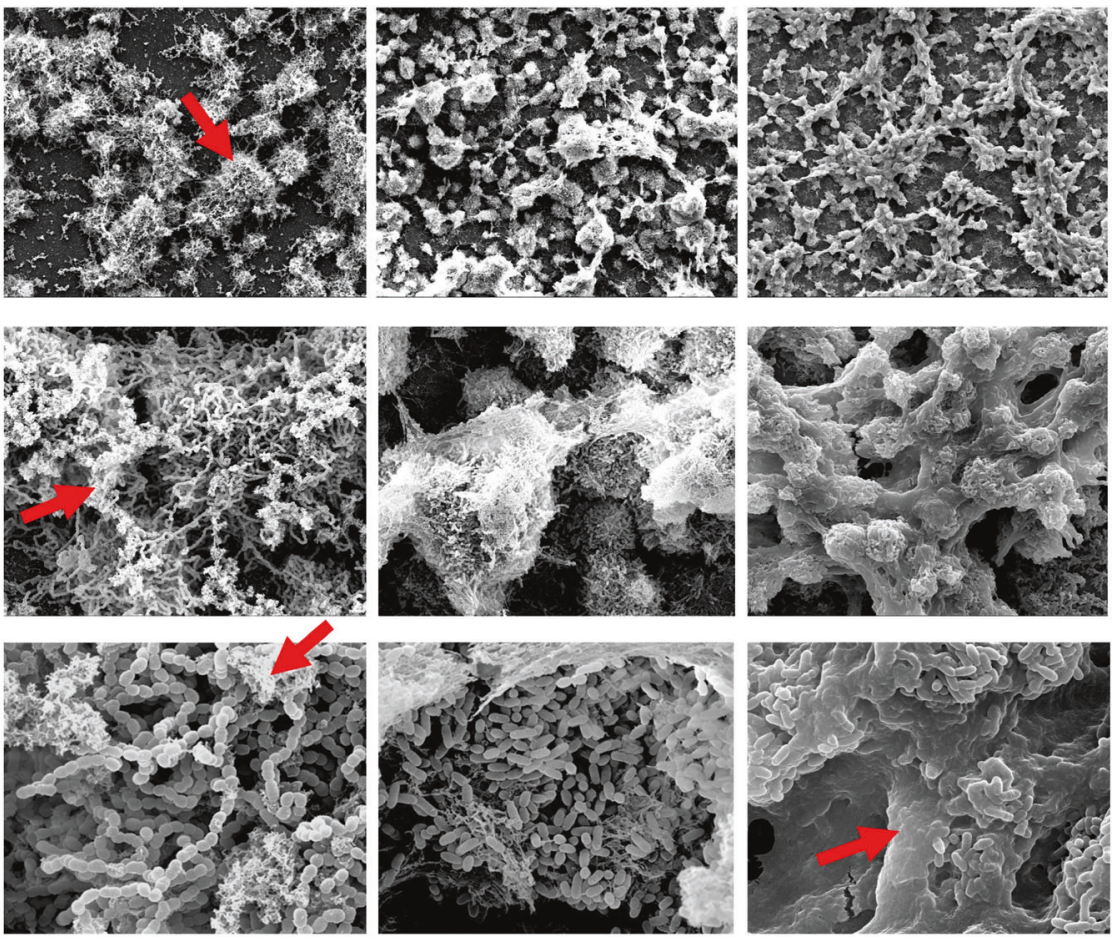

b
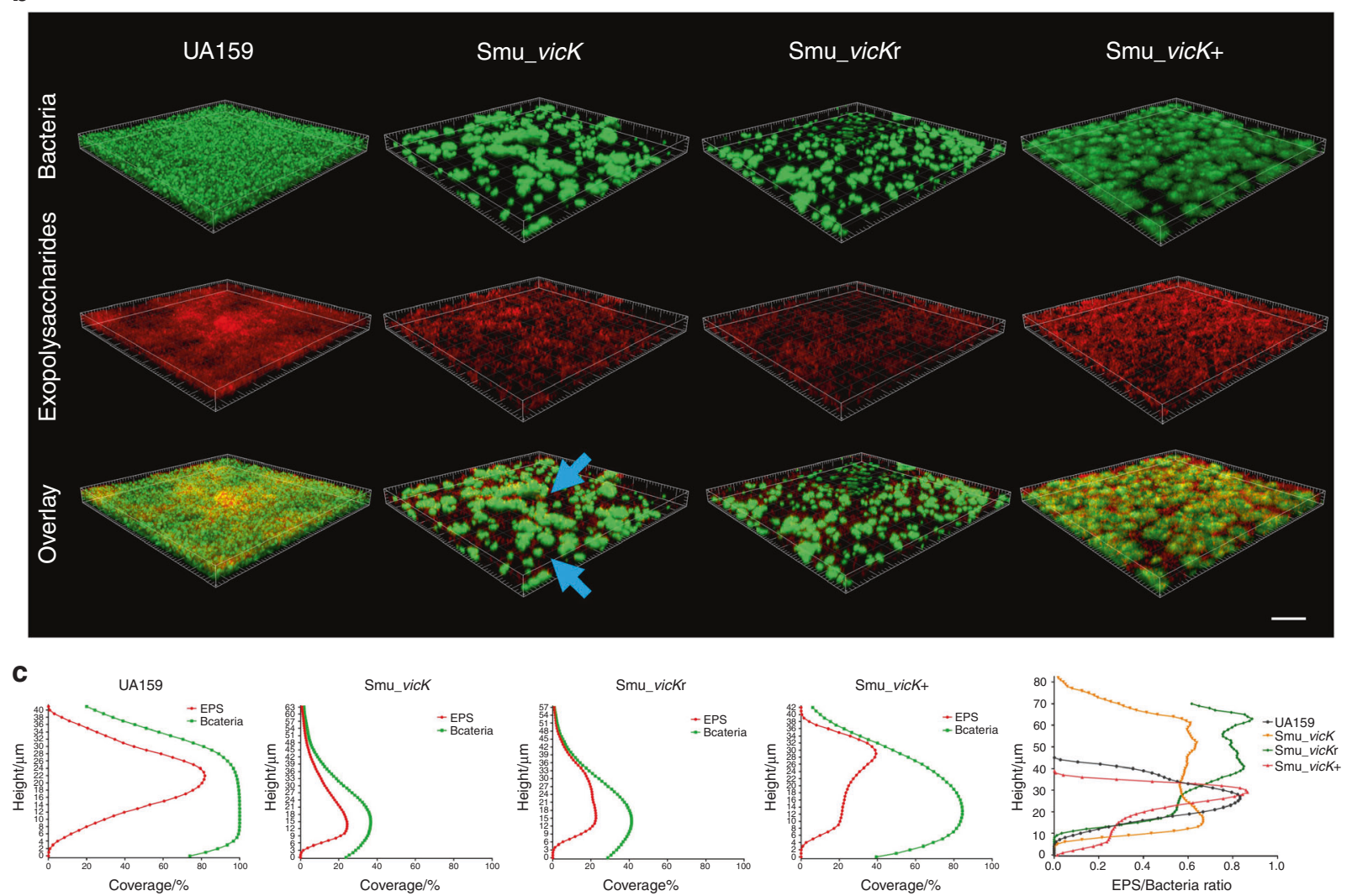

Fig. 2 Analysis of 3-D bacteria-polysaccharides structure in mature biofilms of S. mutans strains. a Biofilm architecture was observed by SEM, in which images were taken at $1000 \times, 5000 \times$, and $20000 \times$ magnifications, respectively (EPS: red arrows). b Representations of glucans distribution and biofilm reconstructions were revealed by CLSM, where EPS stained with Alexa Fluor 647 were red and total bacteria stained with SYTO9 were green. Images were taken at 20x magnification and the scale bar indicated $100 \mu m$. Smu_vicK biofilms showed small clusters of bacterial cells without enough EPS filling the space between them (light blue arrows). c Quantification of EPS and bacteria components, as well as EPS/bacteria ratios at different heights were performed with COMSTAT 
a
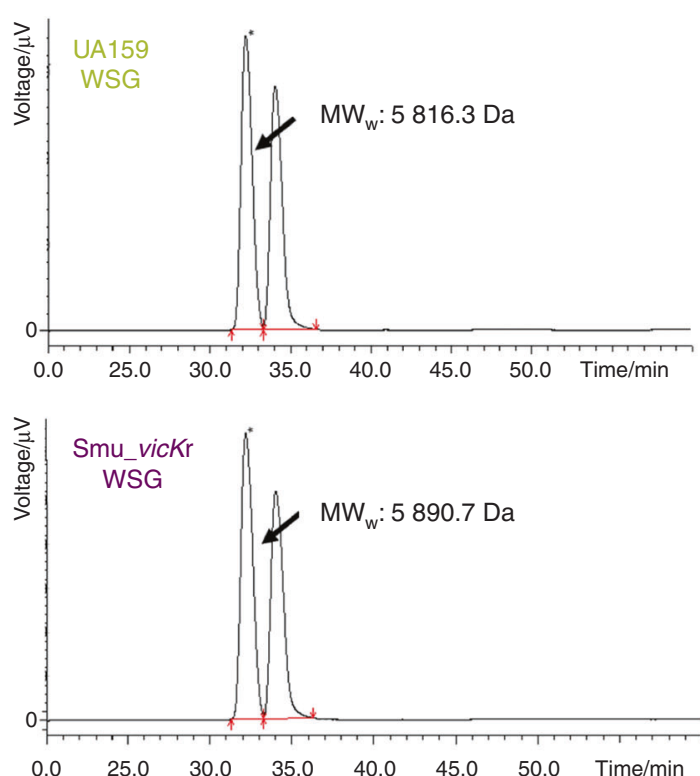

b
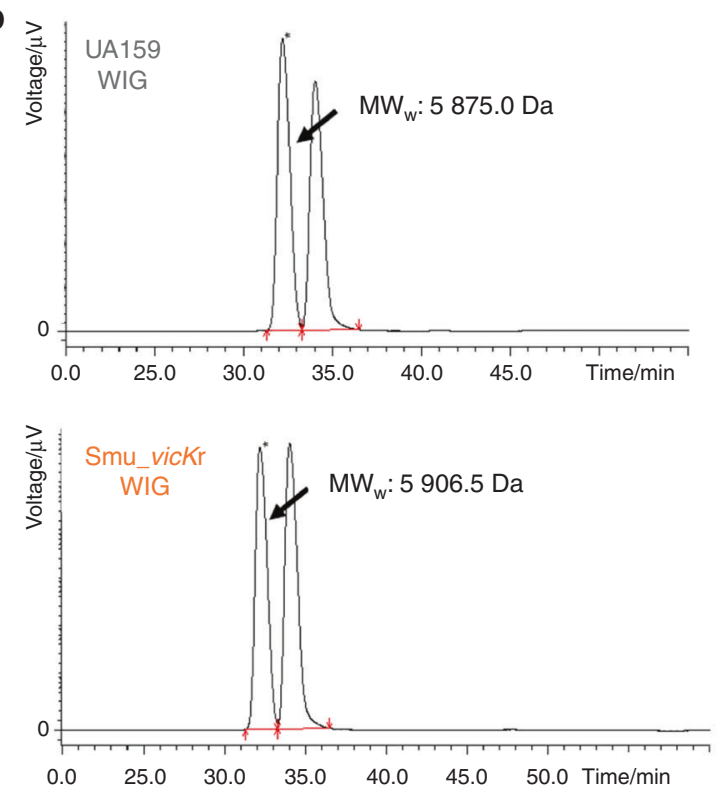

C

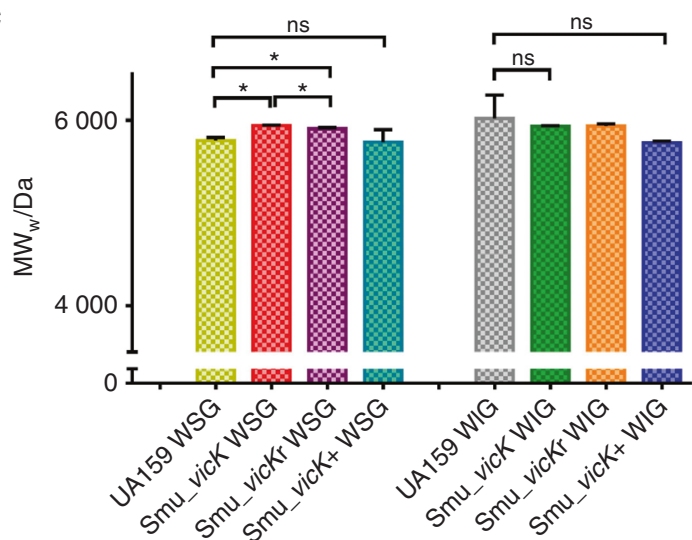

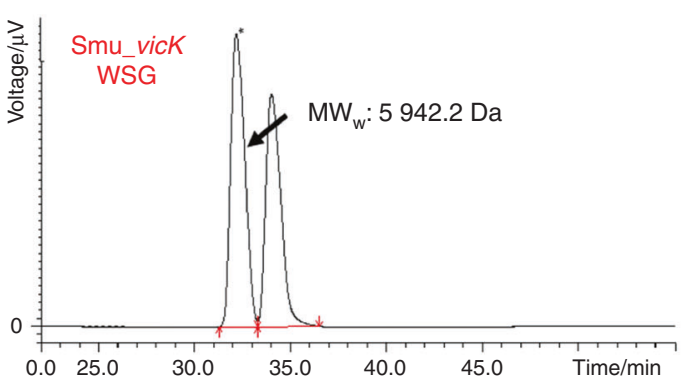
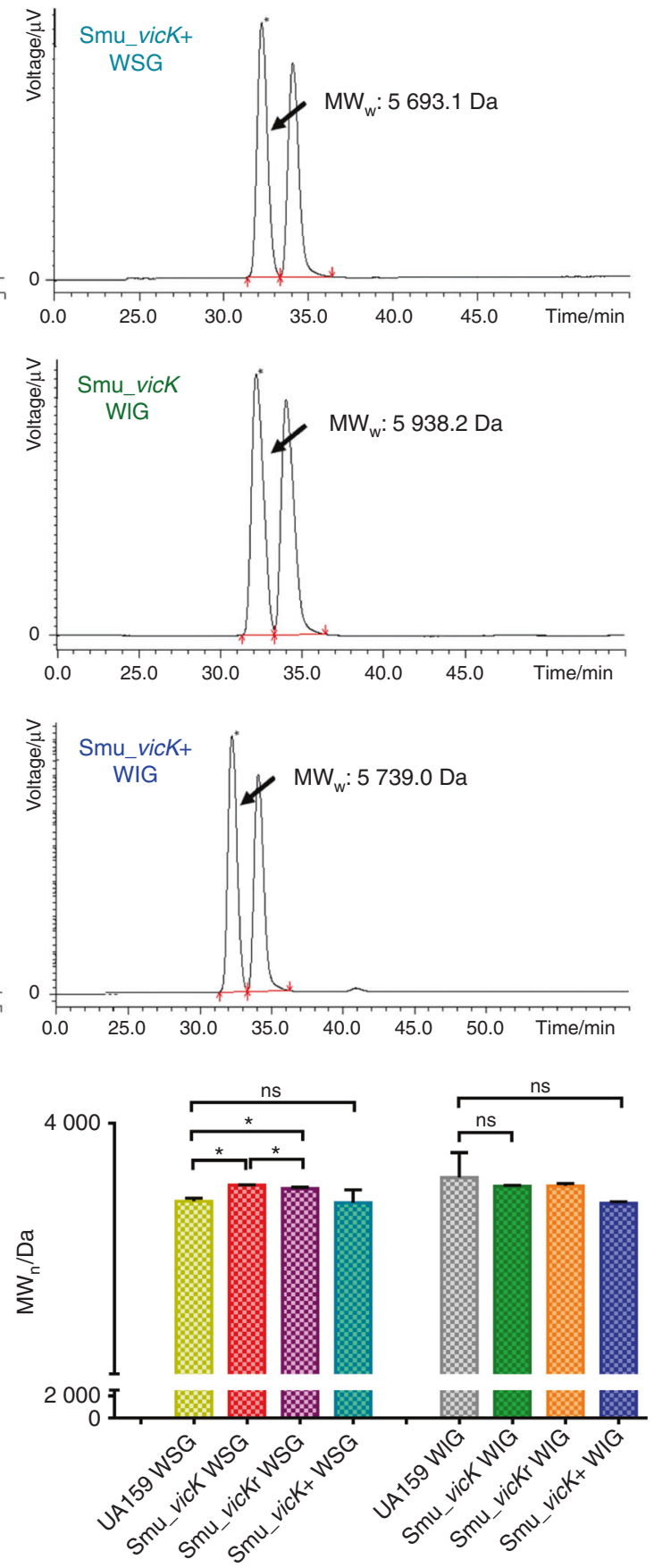

Fig. 3 Molecular weight distributions of EPS were estimated by GPC. The calibration curves were constructed based on the molecular weight of standard dextran and retention time (RT), which were $\operatorname{lgMW}_{\mathrm{w}}=-0.2851 \mathrm{RT}+12.969$ and $\operatorname{lgMW}_{\mathrm{n}}=-0.2666 \mathrm{RT}+12.261$, with $R^{2}=$ 0.9945 and 0.993 9, respectively. a, b Typical GPC chromatograms of polysaccharides from WSGs and WIGs. a Representative MW ${ }_{\mathrm{w}}$ for WSGs. b Representative $\mathrm{MW}_{\mathrm{w}}$ for WIGs. c Distributions of mean $\mathrm{MW}_{\mathrm{w}}$ and $\mathrm{MW}_{\mathrm{n}}$. One-way ANOVA was used to detect the statistical significances. $\left(n=3 ;{ }^{*} P<0.05 ;\right.$ ns no significant difference) 
a
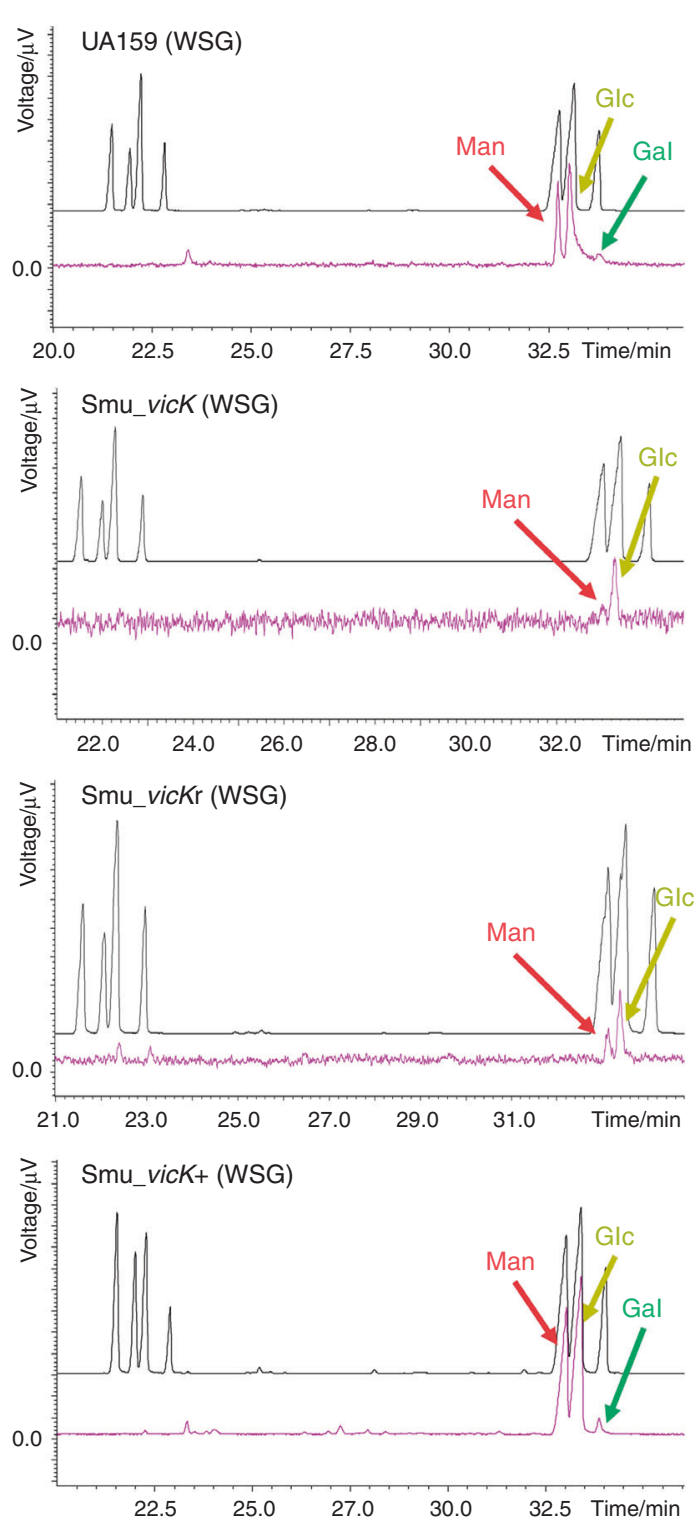

b

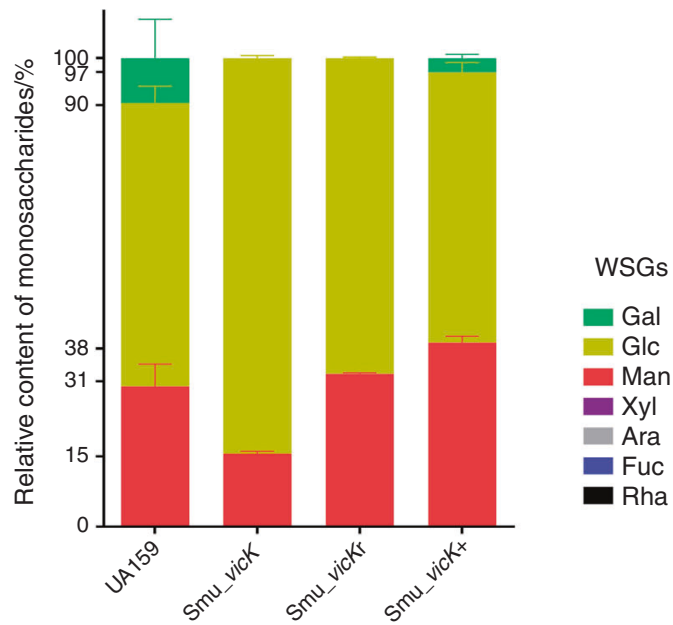

C
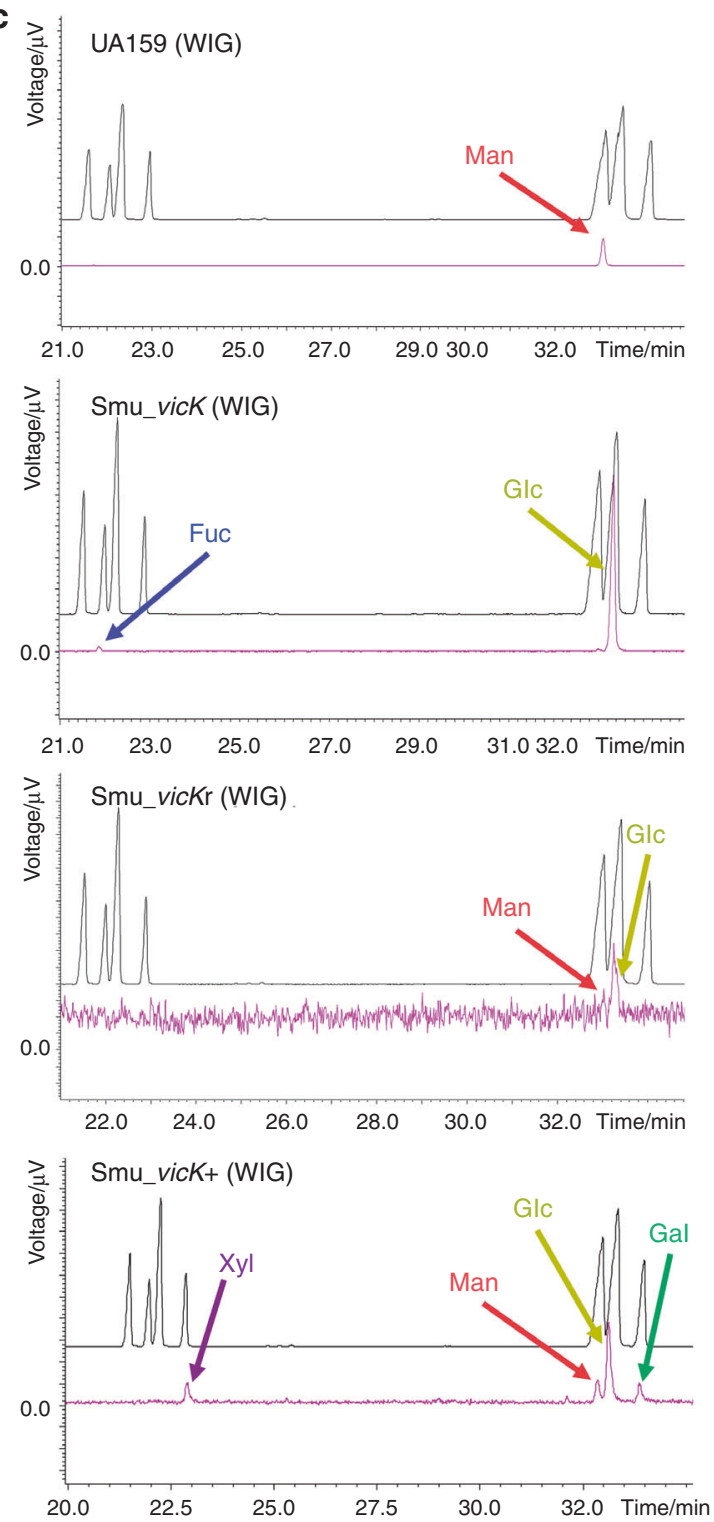

d

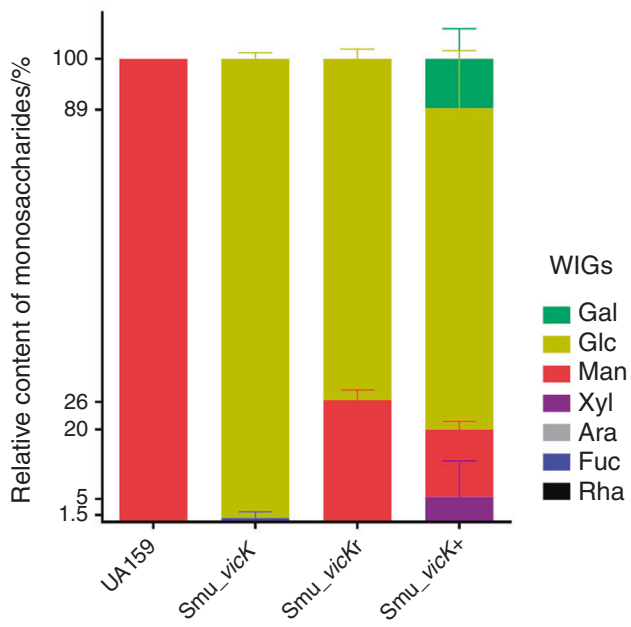

Fig. 4 Monosaccharides of EPS were analyzed by GC-MS. a Typical chromatograms of constituent monosaccharides in WSGs. In these profiles, the black image represented standard samples in sequence: Rha, Fuc, Ara, Xyl, Man, Glc, and Gal, while the pink one represented the experimental samples. b Molar ratios of different monosaccharides in WSGs. $(n=2)$. c Typical chromatograms of constituent monosaccharides in WIGs. d Molar ratios of different monosaccharides in WIGs. $(n=2)$ 
mannose was $29.96 \% \pm 3.33 \%$, glucose was $60.49 \pm 2.53 \%$, and galactose was $9.56 \% \pm 5.86 \%(n=2$, Fig. $4 \mathrm{~b})$. At the same time, WSGs from Smu_vickr biofilms were composed of mannose $(32.62 \% \pm 0.14 \%)$ and glucose $(67.38 \% \pm 0.14 \%)$; WSGs from Smu_vicK+ biofilms were more like those from UA159 (Fig. 4a, b). For WIGs (Fig. 4c, d), UA159 exhibited a 100\% content of mannose and Smu_vick showed a $99.09 \% \pm 0.91 \%$ content of glucose. Molar ratios of Smu_vickr WIGs were $26.41 \% \pm 1.49 \%$ and $73.59 \% \pm 1.49 \%$, for mannose and glucose, respectively. And Smu_vicK+ WIGs seemed to be more complicated consisting of xylose, mannose, glucose and galactose.

Depletion of vick represses cariogenicity of S. mutans Cariogenicity of Smu_vick in vivo was examined by a specific pathogen-free (SPF) rat model. Biofilm overview and EPS distribution were captured by scanning electron microscopy (SEM). In SEM images, Smu_vicK biofilms were sparser with thinner EPS than those formed by UA159 (Fig. 5a). According to the severity and penetration of caries, dental caries was classified into three categories: enamel only $\left(D_{\mathrm{E}}\right)$, slight dentinal $\left(D_{\mathrm{S}}\right)$ and extensive dentinal $\left(D_{\mathrm{X}}\right)$. Smu_vicK group exhibited a significantly increased degree of caries compared to blank group and a significantly decreased degree of caries compared to UA159 group in the $D_{\mathrm{E}}$ and $D_{\mathrm{S}}$ categories (Fig. $5 \mathrm{~b}$ ). However, the severity of $D_{\mathrm{X}}$ was unaffected by depletion of vick gene.

vick alters the expressional levels of virulence-related genes To evaluate effects of vicK gene on signal transduction system and cariogenicity-related genes, the gyrA gene was used as an endogenous reference. As shown in Fig. 5c, transcriptional levels of vicX and gcrR genes were decreased in Smu_vicK when compared to UA159. Simultaneously, $g t f D, f t f$, and $g b p B$ were also downregulated in Smu_vicK. On the contrary, the expressional level of the gtfB gene was significantly increased in Smu_vick. No difference was observed in the expressions of gtfC and $\operatorname{dex} A$.

\section{DISCUSSION}

Biofilms and EPS are inseparably linked. Biofilms develop when microorganisms accumulate on tooth surfaces and secret EPS. ${ }^{27}$ At the same time, EPS initiates the adhesion for first colonizers and is critical for dental biofilms development. ${ }^{28}$ Working together, they can be virulent and cause infectious diseases, like dental caries. ${ }^{29}$ In our study, Smu_vick showed a reduced ability in biofilm accumulation, which was consistent with previous studies that found Smu vick biofilm clumpy and deficient in microcolonies formation. ${ }^{2 \overline{3}, 30}$ Biofilms can act as a barrier and cause resistance against harmful factors, and thicker they are, more protective they are for microbes within. ${ }^{31}$ Thin biofilms like Smu_vick biofilms might be less stable and more easily disrupted, which was consistent with what Senadheera had reported before. ${ }^{23}$ AFM observation of Smu_vick biofilms demonstrated a bumpy topography with spike-like peaks, indicating that knockout of vick gene yielded a thin biofilm with less EPS. Biofilm development is a complex process including cell to cell adhesion. Physical or molecular interactions are involved in this process. ${ }^{32}$ It also has been reported that the surface roughness does not necessarily affect bacterial adhesion. ${ }^{23}$ The limitation of this study was that we did not measure the surface roughness of a substratum surface but the surface roughness of the biofilm itself. Interestingly, Smu_vicK biofilms were thicker than those of UA159 as shown in Fig. 2c, but they seemed sparser and with less EPS (Fig. 2b). In present study, the biofilm surface roughness and adhesion force of Smu_vick biofilms were declined, indicating biofilm surface roughness and adhesion force may be reflections of EPS production in S. mutans, which also warranted further investigations. $^{23,33}$ Similar results came out in SEM and CLSM observations. It was worth mentioning that EPS of Smu_vicK biofilm did overlap with the microcolonies but it was few. Previous reports also showed that the vick-deficient strain had a drastically reduced glycolytic rates. ${ }^{33}$

Indeed, construction of the vick complementary strain was limited in this study, where vick expression was slightly higher than Smu_vick but not close to UA159 level (Fig. S1). As a result, Smu_vicKr biofilms were thin and more like Smu_vicK biofilms. One possible explanation was that the introduction of an exogenous plasmid vector might interfere with the intracellular homeostasis and growth of the mutants, which has been reported in our previous study. ${ }^{16}$ Although Smu_vicKr biofilms show less biomass than the one of Smu_vicK biofilms, the phenotypes of roughness and adhesive forces in the Smu_vicKr biofilms were partially restored when compared to those of UA159 biofilms. Smu_vicK+ biofilms did exhibit a slight increase in biomass or EPS when compared to those of UA159. We speculated that the difference was not that evident because of the introduction of the plasmid. ${ }^{16}$ Biofilms of Smu_vicK+ were not as mature as UA159 in Fig. 2, but it also showed EPS/bacteria ratios of Smu_vicK+ biofilms were higher than those of UA159. One possible explanation for Smu_vicK+ biofilms not as mature as UA159 was that Smu_vicK+ biofilms had larger microorganism clusters and more EPS.

Taken together, deletion of vick gene not only impaired biofilm formation, but also repressed EPS production. EPS can encapsulate microbial communities and provide enough attachment sites for them to form structured biofilms, contributing to biofilm lifestyle and virulence. ${ }^{34}$ In addition to EPS, bacterial aggregation is also an essential part in biofilm formation. ${ }^{23}$ We found a rough bacterial aggregation of Smu_vicK in SEM, an evidently longer chain length and a larger colony of Smu_vicK+ (Fig. 2 and Fig. S2). Decreased EPS and aberrant biofilm might result in alteration of cariogenicity of S. mutans.

Exopolysaccharides is part of macromolecules within extracellular matrix. Both WSGs and WIGs are of great significance. WSGs is able to provide energy source; WIGs can provide protective shelters for the biofilms and modulate environmental acidification. ${ }^{35-37}$ Targeting EPS is a promising approach in biofilm control and recent studies have declared kinds of nanoparticles disrupting biofilm microenvironment, which allows disruption of the matrix. ${ }^{38-40}$ Targeted therapy for dental caries is becoming more popular, ${ }^{41}$ so a thorough understanding of the targeted EPS is a keystone. Structural features of polysaccharides like molecular weight may play an important role in various biological activities. ${ }^{42,43}$ For example, a high-molecular-weight polysaccharide fraction from Sparassis latifolia is recognized to account for a limited biological activity because it makes the fungus difficult to adhere or penetrate cell membranes. ${ }^{44,45}$ Combined with the data we got, it was speculated that the upregulation of molar mass in Smu_vicK EPS might be relevant to its impairment. Molecular weight of polysaccharides can be influenced by glycoside linkage, which is attributed to the linkage cleavage during the enzymatic degradation. ${ }^{46}$ Fructosyltransferase (Ftf, coded by $\mathrm{ftf}$ ) converts sucrose into a predominantly $\beta 2,1$-linked fructan polymer. ${ }^{47}$ Glucosyltransferases (Gtfs, encoded by $g t f B / C / D$ genes) are also linkage-related, in which GtfB synthesizes a1,3-glycosidic linkages of WIGs and GtfD synthesizes a1,6-glycosidic linkages of WSGs. ${ }^{48}$ We assumed the altered expressional levels of $f t f$ and $g t f B / D$ genes in Smu_vicK (Fig. 5c) probably induced glycoside linkage alternation and subsequent molecular weight of polysaccharides. Further studies like methylation analysis will provide the information of the glycosidic linkages of EPS. ${ }^{49}$ Monosaccharides are essential substrates to exopolysaccharide chains, and different compositions of them can make different biofilm matrix. ${ }^{50,51}$ Constituent ratio of glucose experienced evident increasing in EPS of Smu_vicK biofilms, indicating a rising capability of Smu_vicK to synthesize glucose. It was speculated that polysaccharides of Smu_vick biofilms were heteropolysaccharides containing glucose, and the 
a
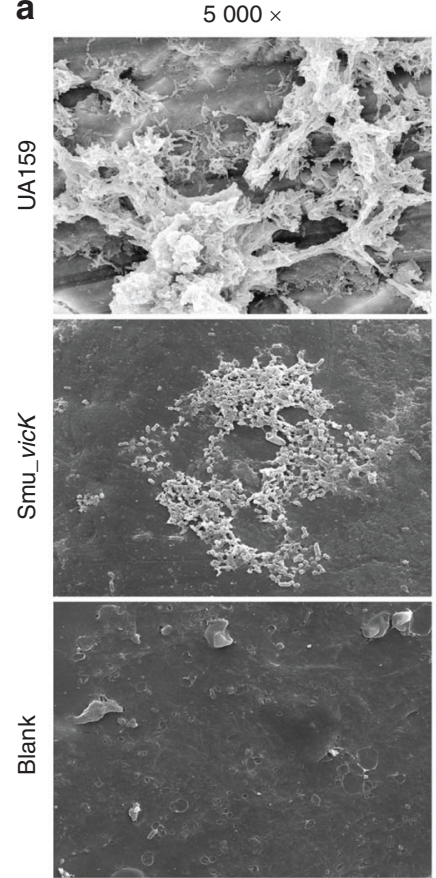

b

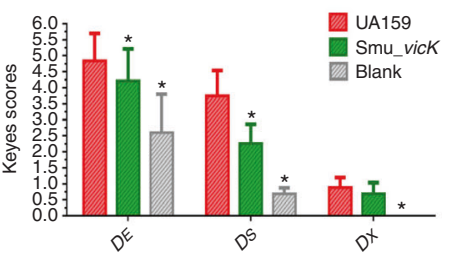

$10000 \times$

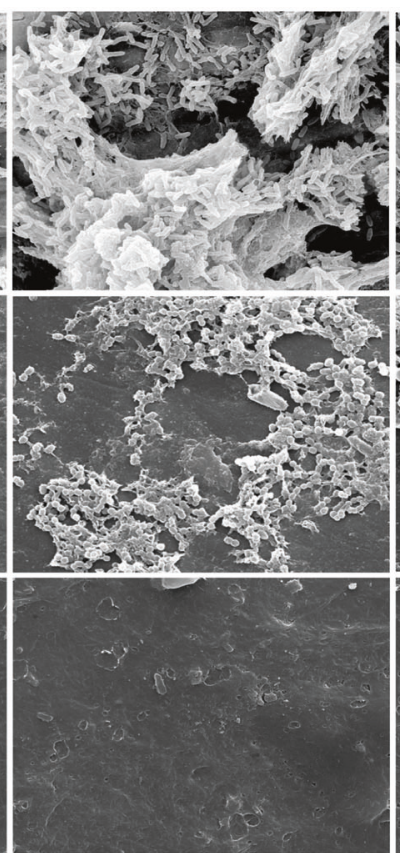

C

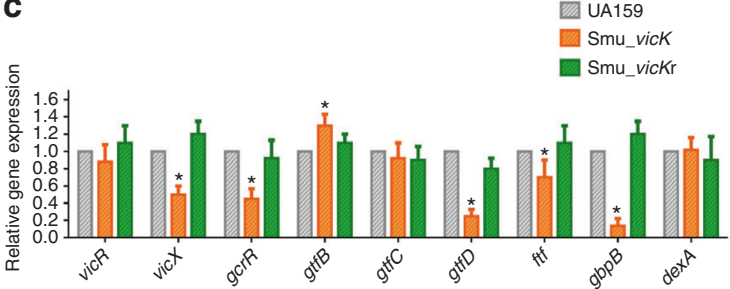

d

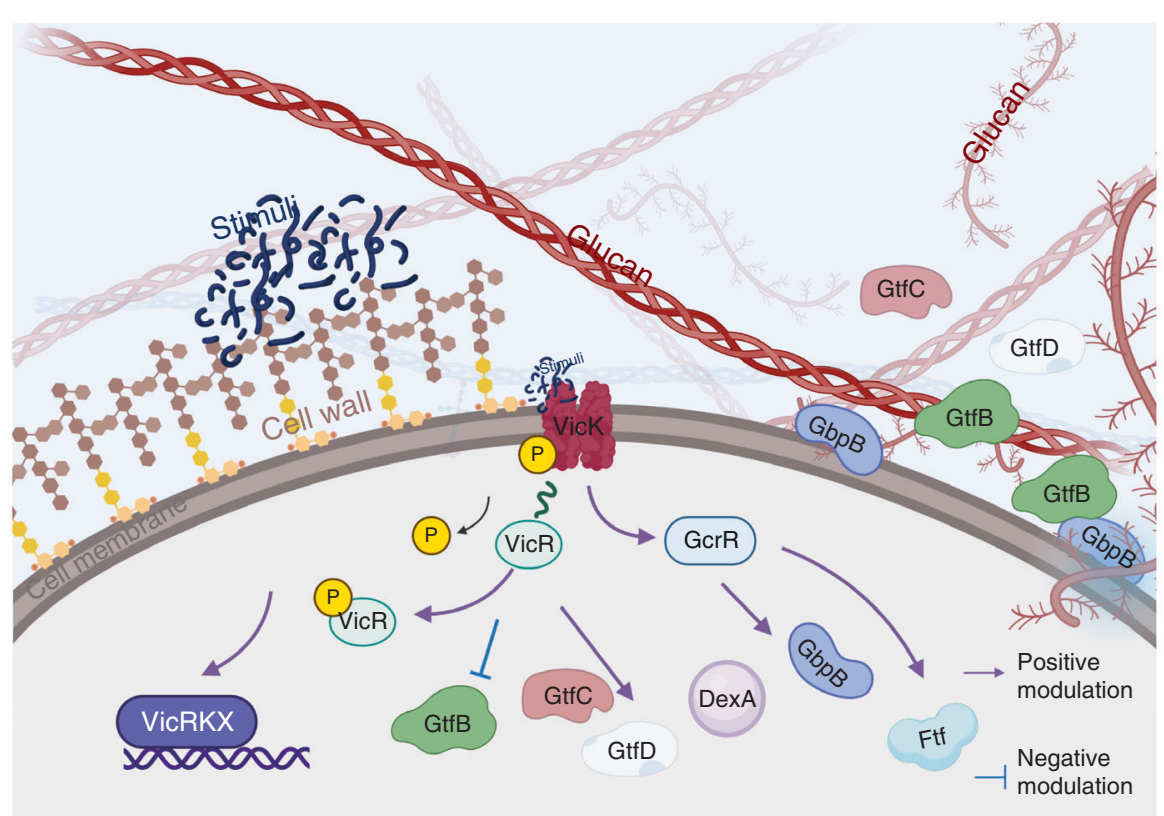

Fig. 5 Influences of the deletion of vicK gene on cariogenicity and associated genes. a Dental plaques on mandibular molars in rats were shown by SEM with pictures taken at $1000 \times, 5000 \times$, and $20000 \times$ magnifications. $\mathbf{b}$ The degrees of caries were divided into three categories by depth of penetration: $D_{\mathrm{E}}, D_{\mathrm{S}}$, and $D_{\mathrm{X}}$ according to the modified Keyes scores. Unpaired $t$ test was used to detect the statistical significances. $\left(n=10 ;{ }^{*} P<0.05\right)$. c Expressional levels of EPS-correlated genes were measured by qRT-PCR, where UA159 was taken as the control group and the gyrA gene was used as an internal standard (not shown). One-way ANOVA was used to detect the statistical significances. $\left(n=3 ;{ }^{*} P<0.05\right)$. d The schematic illustration of regulations conducted by vicK on the EPS metabolism of S. mutans. Created with BioRender.com. Adapted from “ECM (Extracellular Matrix)", by BioRender.com (2021). Retrieved from https://app.biorender.com/biorender-templates. After being activated by environmental stimuli, Vick has vital influences on the expression of genes correlated with EPS, whose encoding proteins promote the synthesis of EPS, subsequently enhances the biofilm virulence 
side chains might be composed of mannose or fucose, which can be confirmed by connection mode analysis in further studies. ${ }^{52}$ EPS is a vital attribute for the bacterial community structuring and contributes a lot in biofilm lifecycle and subsequent caries development. ${ }^{53}$ We regarded that vick gene and microcosmic structures of EPS were closely related, which might have a relationship with cariogenicity of $S$. mutans biofilms.

A dysregulation of biofilm formation and decreased cariogenicity of Smu_vick in vivo could be seen in this study. Evidently, these results were consistent with the results in vitro. However, Senadheera had found that in low-cariogenic diet feed rats, the development of dentinal lesions was not significantly altered in Smu_vick group compared with UA159, ${ }^{23}$ which was different from what we found when we fed the rats a normal diet with drinking water containing $2 \%$ sucrose. S. mutans can make effective use of dietary sucrose for EPS production, using $\mathrm{GtfB} / \mathrm{C} /$ D. ${ }^{54,55}$ Both the diet and the nature of EPS can determine the diffusion properties of plaque and cariogenicity of S. mutans. VicK acts as a receptor of extracellular stimuli, ${ }^{56}$ and when it was deleted, the capacity of bacterial cell to get transduction signals might be impaired. On the other hand, expressions of $g t f B / C / D$ genes could also be affected by vick as previously reported, ${ }^{23}$ which are associated with sucrose. One possible explanation of this difference was that Smu_vicK had defects in biofilm formation while UA159 formed more compact and cariogenic biofilms under high-cariogenic circumstances. In addition, it has been found that the vick-deficient strain produced less lactic acid, ${ }^{33}$ which might partially contribute to the low cariogenicity of the Smu_vick biofilm. These data suggested that we should attach more importance to vick gene and dietary sucrose.

In present study, vicK gene positively modulated vicR/X and $g c r R$. The vicR/KJX gene are in the same operon: the vic operon, ${ }^{57}$ so deletion of vick gene might influence the transcriptional levels of vicR/X. GcrR (also named CovR, coded by gcrR gene) is known as an orphan response regulator in $S$. mutans, with no exclusively linked cognate histidine kinase. ${ }^{58}$ There is a co-regulation between GcrR and VicRK, ${ }^{59,60}$ in which, like VicR, GcrR can also directly binds to the promoter regions of some genes, but opposite to VicR, it acts like a repressor. ${ }^{61-63}$ And Vick can activate GcrR by phosphorylating it. ${ }^{64,65}$ The declined expressional level of $g c r R$ in this study was not in coincidence with the opposite mechanism between VicR and GcrR. What's more, we found a significant decrease of GcrR protein in Smu_vicK (Fig. S3). Although there was also a decrease in expressional level of VicR protein, it seemed relatively stable when compared with GcrR (Fig. S3). On one hand, it has been reported that deletion of vick gene does not have a significant influence on the ratio of phosphorylated VicR to unphosphorylated VicR. ${ }^{66}$ On the other hand, it has also been authenticated that neither dimerization nor phosphorylation status of GcrR are essential for its activity. ${ }^{59}$ As a result, we speculated that VicK might also influence the expressional level of GcrR in some way to balance the regulatory system, which need further studies. Simultaneously, gtfD, ftf and $g b p B$ were also downregulated in Smu_vick as previously reported. ${ }^{23}$ The less-cariogenic biofilm with poorer EPS generated by Smu_vicK might be resulted from the defect of $f t f$ and $g b p B$ genes because Ftf and GbpB (encoded by $g b p B$ ) should have enhanced the cariogenic potential of $S$. mutans by extending acidification or working with Gtfs. ${ }^{67-69}$ Unexpectedly, the expressional level of gtfB gene was significantly increased and that of gtfC was unchanged in Smu_vicK, which were in contrast with a previous observation showing a 3.0 and 1.4-fold downregulation of $\mathrm{gtfB} / \mathrm{C}$ in Smu_vicK at mid-log phase grown in brain heart infusion (BHI) medium. ${ }^{70}$ We considered the variation in nutrients was responsible for the differences. ${ }^{71,72}$ It was interesting that the increase of gtfB did not appear to provide a mechanically integrated and stable extracellular insoluble matrix for Smu_vicK biofilms. This phenomenon might be explained by the extreme decrease of $g t f D$, whose products are supposed to serve as primers for GtfB activity. ${ }^{73}$ In S. mutans, Gtfs perform a concerted action to produce glucans and affect EPS. ${ }^{7475}$ It was once discussed that an optimal GtfB/GtfC/GtfD ratio was necessary for appropriate colonization of $S$. mutans in vitro. ${ }^{76}$ We speculated that the expression of vick gene mostly influenced EPS-synthesis genes in a positive way because the expressional level of $\operatorname{dex} A$ gene was not changed in Smu_vick. A schematic illustration was shown in Fig. 5d. Understanding the mechanisms involved in biofilm-associated gene expressions is crucial for us to develop new therapeutic strategies. It has been reported that expressional levels of vicR/gcrR genes, key regulators to EPS-synthesis, are maximal in S. mutans which are at mid-exponential phase. ${ }^{77}$ However, the gene expressions of planktonic cultures may not reflect the expressions of biofilm grown cells, which needs further investigations.

Carbohydrate metabolism is necessary to viability and virulence of S. mutans, in which production of lactic acid, acid tolerance and production of EPS are all essential. ${ }^{78}$ S. mutans is able to produce lactic acid as a metabolic product, which induces lower $\mathrm{pH}$ value in environment, as well as the enamel erosion and dental caries. The acid tolerance response becomes a key survival mechanism for the acid stress adaption. ${ }^{79}$ Senadheera et al. had studied the effects of $S$. mutans vicK gene on acidogenicity and aciduricity in the presence of glucose. They found vick deletion mutant produced less lactic acid with its acid tolerance enhanced. ${ }^{33}$ However, the effects of $S$. mutans vick gene on EPS production in the presence of sucrose were not fully elucidated. So, in current study, we provided evidences proving the modulation of vick gene on EPS of $S$. mutans biofilm. The vicK gene regulated biofilm characteristics, including synthesis as well as component and structural modifications of EPS. These data illustrated a significant situation where vick was a promising governor, being involved in cariogenicity of $S$. mutans through EPS metabolism. We hypothesized vick gene enhanced transcriptional levels of EPS-induced genes in a signal transduction cascade and it could increase the production of EPS, change the microcosmic features of EPS as well as strengthen the virulence of $S$. mutans.

\section{MATERIALS AND METHODS}

Strains and bacterial culture conditions

S. mutans standard strain, ${ }^{36}$ UA159 (Table 1) was provided by State Key Laboratory of Oral Diseases, West China School of Stomatology, Sichuan University. An erythromycin resistance cassette was utilized to knock out the vicK gene in UA159 chromosome by polymerase chain reaction (PCR) ligation mutagenesis to get Smu_vicK. ${ }^{23,80}$ Recombinant pDL278 plasmids which contained the vick gene coding region were introduced into Smu_vicK and UA159 cells respectively to construct the vicK complementary strain (Smu_vicKr) and the vicK overexpression strain $\left(S m u \_v i c K+\right) .{ }^{81}$ The expressions of vick gene in the mutants were verified by quantitative real-time PCR (qRT-PCR) (Fig. S1). Conventionally, UA159, Smu_vicK, Smu_vicKr, and Smu_vicK+ were cultured into mid-exponential phase in BHI medium (Becton, Dickinson and Company, Sparks, MD 21152 USA) at $37^{\circ} \mathrm{C}$, anaerobically, with appropriate antibiotics when necessary. The achieved final concentrations of antibiotics were $10 \mu \mathrm{g} \cdot \mathrm{mL}^{-1}$ erythromycin for Smu_vicK, $1000 \mu \mathrm{g} \cdot \mathrm{mL}^{-1}$ spectinomycin for Smu_vicK+, $10 \mu \mathrm{g} \cdot \mathrm{mL}^{-1}$ erythromycin and $1000 \mu \mathrm{g} \cdot \mathrm{mL}^{-1}$ spectinomycin for Smu_vickr.

\section{Biofilm formation}

Strains were grown to mid-exponential phase at an $\mathrm{OD}_{600 \mathrm{~nm}}$ of 0.5 and then were diluted 1:100 into $\mathrm{BHI}$ medium containing $1 \%(\mathrm{w} / \mathrm{v})$ sucrose (BHIS). Biofilms were formed at $37^{\circ} \mathrm{C}$, anaerobically for $24 \mathrm{~h}$.

\section{CV assay}

Biofilm mass was quantified by CV assay as previously described. ${ }^{82}$ Briefly, biofilms formed on a polystyrene surface in a $96-$-well plate were washed by sterilized deionized distilled 
Table 1. Bacterial strains and plasmid used in this study

Strain or plasmid Description

UA159 S. mutans UA159

Smu_vicK

Smu_vicKr

Smu_vicK +

pDL278

S. mutans UA159 mutans UA159 with in-frame replacement with an erythromycin cassette

Smu_vick transformed with pDL278 encoding vick gene

S. mutans UA159 transformed with pDL278 encoding vicK gene

Escherichia coli-Streptococcus shuttle vector and expression plasmid (spectinomycin)
Source of reference

ATCC 700610

This study

This study

This study

Gifted by Dr. Huichun Dong, Institute of Microbiology, Chinese Academy of Sciences water three times to remove planktonic bacteria cells, then were stained with $0.1 \%(\mathrm{w} / \mathrm{v}) \mathrm{CV}$ for $15 \mathrm{~min}$. The stained biofilms were gently rinsed again and air dried, followed by an addition of $33 \%$ (v/v) glacial acetic acid to solubilize the dye. At last, the optical density was read at $575 \mathrm{~nm}$ (SpectraMax ${ }^{\oplus}$ iD5, Molecular Devices, San Jose, California, USA).

\section{Atomic force microscopy}

Surface topography and adhesion force of the biofilms were analyzed by AFM as conducted previously. ${ }^{83,84}$ Biofilms were incubated in a 24 -well plate for $24 \mathrm{~h}$ with round, sterile glass slides, then the slides were washed by sterilized deionized distilled water and dried for two minutes in air. Shimadzu SPM-9700 system (SHIMADZU, Kyoto, Japan) was conducted in the contact mode using a specialized probe (HYDRA-ALL-G-20, APPNANO, USA) for the measurements.

\section{Scanning electron microscopy}

Architecture of the biofilms was measured by SEM (Inspect F, FEl, Eindhoven, Holland) using round, sterile glass slides. ${ }^{85}$ The biofilms on slides were washed by sterilized deionized distilled water then fixed with $2.5 \%(\mathrm{v} / \mathrm{v})$ glutaraldehyde overnight. Next day, they experienced a sequential dehydration in ethanol solutions and then were prepared for imaging. ${ }^{86}$ The specimens were examined at $1000 \times, 5000 \times$, and $20000 \times$ magnifications, and representative pictures are shown.

\section{Confocal laser scanning microscopy}

Three-dimensional (3-D) structure and EPS distribution of the biofilms were observed by CLSM (Olympus FV1000, Japan). ${ }^{87}$ $1 \mu \mathrm{mol} \cdot \mathrm{L}^{-1}$ Alexa Fluor 647 (Invitrogen, Eugene, Oregon, USA) were added into $2 \mathrm{~mL}$ BHIS with $20 \mu \mathrm{L}$ bacterial cultures before biofilm incubation to label dextran conjugate. Biofilms were incubated in a 24-well plate for $24 \mathrm{~h}$ in dark, with round, sterile glass slides. After biofilm formation, nucleic acid of $S$. mutans cells were labeled with $2.5 \mu \mathrm{mol} \cdot \mathrm{L}^{-1}$ SYTO9 (Invitrogen, Eugene, Oregon, USA). Microscopic observations were performed with a $20 \times$ objective lens, and 3-D images were reconstructed by Imaris 7.0.0 software (Bitplane, Zürich, Switzerland). Calculation of EPS/ bacteria biomass were performed with COMSTAT.

\section{Exopolysaccharides assessment}

Molecular weights of EPS were analyzed by gel permeation chromatography (GPC) and monosaccharide composites were analyzed by gas chromatography-mass spectrometry (GC-MS). Both of WSGs and WIGs derived from $24 \mathrm{~h}$ biofilms were isolated and purified for GPC and GC-MS analysis as reported before. ${ }^{16}$ In short, biofilms suspended by sterilized deionized distilled water were centrifuged to separate WSGs solution and other insoluble precipitates containing WIGs. The insoluble precipitates were dissolved in $1 \mathrm{~mol} \cdot \mathrm{L}^{-1} \mathrm{NaOH}$ solution and then filtered through a $0.22 \mu \mathrm{m}$ filter (Merck Millipore Ltd. Tullagreen, Carrigtwohill, Co. Cork, IRL) to get WIGs solution. Then, $20 \%(\mathrm{w} / \mathrm{v})$ trichloroacetic acid was added into the solution mentioned above for protein precipitations. After that, solutions were dialyzed (cellulose membrane with a molecular weight cut-off of 500-1 $000 \mathrm{Da}$ ) for further purification and were lyophilized afterwards. The lyophilized powder was used for GPC and GC-MS. ${ }^{88}$ For GPC analysis, sample polysaccharides in a final concentration of $5 \mathrm{mg}$ $\mathrm{mL}^{-1}$ and standard solutions of dextran in different molecular weights (MW; MW 1 152, 5 200, 11 600, 148 000, 273 000, 410 $000 \mathrm{Da}$; Waters, Massachusetts, USA) were loaded in a high performance liquid chromatograph (LC-10A, SHIMADZU, Japan). ${ }^{51}$ For GC-MS analysis (QP 2010 Plus, SHIMADZU, Japan), samples were further acetylated and the compositions of EPS were identified with standard monosaccharide substances (Sigma, San Francisco, CA, USA), which included rhamnose (Rha), fucose (Fuc), arabinose (Ara), xylose (Xyl), mannose (Man), glucose $(\mathrm{Glc})$, galactose $(\mathrm{Gal}) .^{89}$

\section{Cariogenicity ability in vivo}

Effects of vick gene on cariogenicity of S. mutans in vivo were assessed in a SPF rat model..$^{90}$ This study was approved by the Ethics Committee of West China Hospital of Stomatology, Sichuan University (NO: WCHSIRB-D-2019-144), and conducted in IVC Experimental Animal Center of Public Health, Sichuan University (Chengdu, China). Thirty caries-susceptible, Osborne-Mendel rats (15 male rats and 15 female rats), aged 14 day old (Dashuo Company, Chengdu, China) were included and randomly assigned into 3 groups: Blank (the negative control), UA159 (the positive control), and Smu_vicK (the experimental group). Posterior to endogenous Streptococci test, ${ }^{91}$ on days $23-30$, rats were infected orally, daily for one week, using $200 \mu \mathrm{L}$ bacterial suspensions that comprised UA159 or Smu_vicK, respectively. Bacterial suspensions were collected when strains grew to midexponential phase at the same $\mathrm{OD}_{600 \mathrm{~nm}} 0.4$. All rats received drinking water containing $2 \%$ sucrose as well as a normal diet, and animals were sacrificed on day 50 . Then, their lower jaws were excised for plaque observation using SEM and caries level determination using modified Keyes score. ${ }^{92}$

\section{Gene expression assay}

S. mutans cells, which included UA159, Smu_vicK and Smu_vicKr, were collected at mid-exponential phase in BHIS by centrifugation at $4000 \mathrm{r} \cdot \mathrm{min}^{-1}$ for $15 \mathrm{~min}$ (Thermo Fisher SCIENTIFIC, Germany). Gene expression was analyzed by qRT-PCR. Total RNAs of $S$. mutans cells were isolated and purified using MasterPure ${ }^{\mathrm{TM}}$ Complete DNA and RNA Purification kit (Lucigen Corporation, Wisconsin, USA) according to the instructions. Concentration and purity of total RNAs were assessed by NanoDrop One ${ }^{C}$ Microvolume UV--Vis Spectrophotometer (Thermo Scientific, Waltham, MA, USA). After that, RNAs were reverse transcribed into CDNA using PrimeScript ${ }^{\mathrm{TM}}$ RT reagent kit with gDNA eraser (TAKARA BIO INC. Kusatsu, Shiga, Japan). Optimal sample or primer concentrations were followed by the instruction of TB Green ${ }^{\mathrm{TM}}$ Premix Ex $\mathrm{Taq}^{\mathrm{TM}}$ II kit (TAKARA BIO INC. Kusatsu, Shiga, Japan). In brief, each $20 \mu \mathrm{L}$ reaction mixture included $10 \mu \mathrm{L}$ of TB Green ${ }^{\mathrm{TM}}$ Premix Ex $\mathrm{Taq}^{\mathrm{TM}} \mathrm{II}, 0.8 \mu \mathrm{L}$ of $10 \mu \mathrm{mol} \cdot \mathrm{L}^{-1}$ PCR Forward Primer, $0.8 \mu \mathrm{L}$ of 
Table 2. List of the primers used in this study

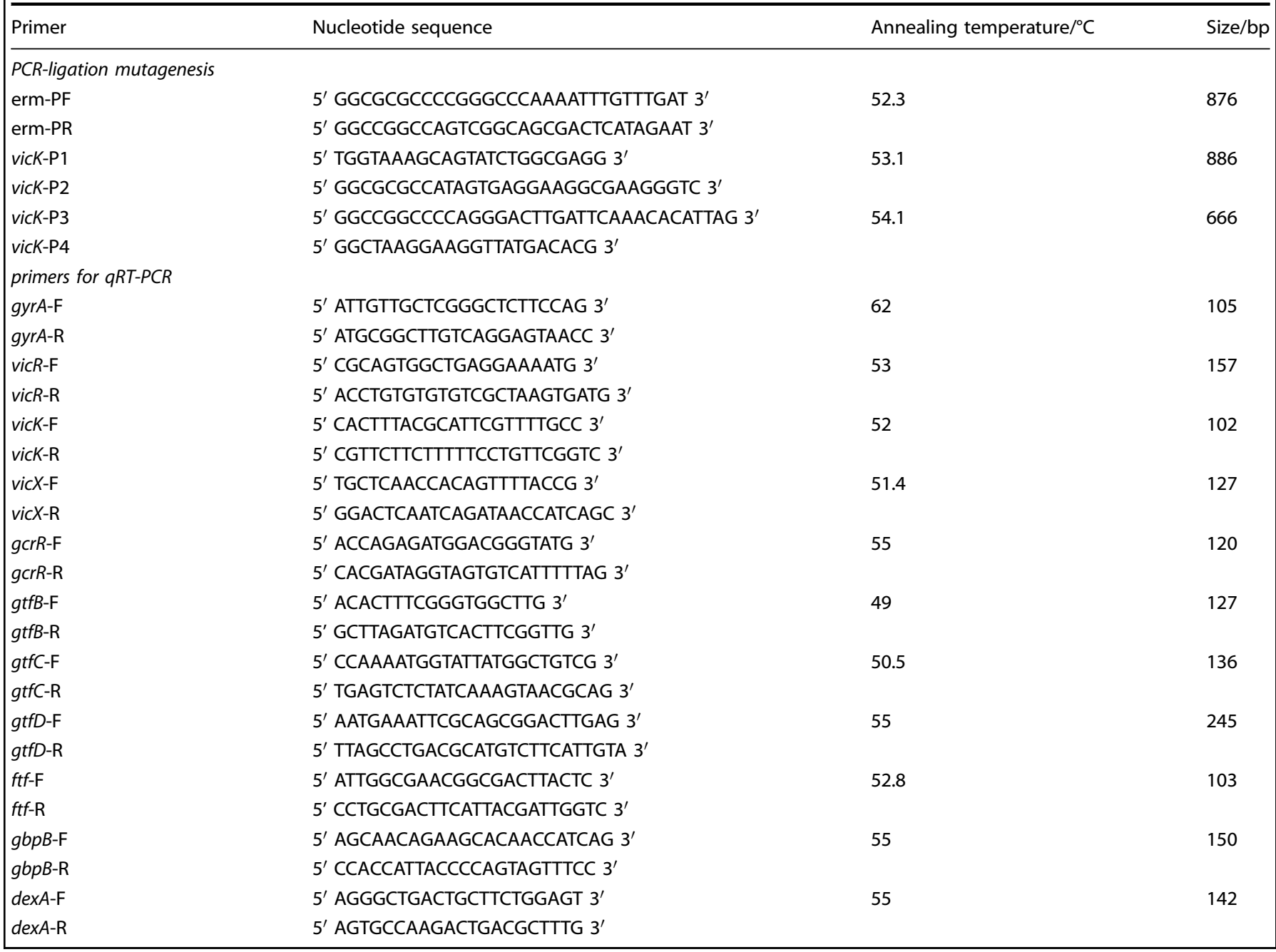

$10 \mu \mathrm{mol} \cdot \mathrm{L}^{-1}$ PCR Reverse Primer, $2.0 \mu \mathrm{L}$ of template CDNA and $6.4 \mu \mathrm{L}$ of deionized water. And qRT-PCR was conducted in a LightCycler 480 System (Roche, Basel, Switzerland) according to the instruction above. ${ }^{93}$ The expressional levels of $g y r A, v i c R / X$, $g c r R, g t f B / C / D, f t f, g b p B$ and $\operatorname{dex} A$ genes were evaluated. All primers for QRT-PCR were designed based on the sequence of the S. mutans UA159 genome according to previous studies and obtained commercially (Sangon Biotech, Shanghai, China). ${ }^{23,88}$ They were listed in Table 2. Gene expressions were calculated by the $2^{-\Delta \Delta C T}$ method in which $P<0.05$ were thought to be significantly different. $^{94}$

Statistical analysis

Statistical analysis was executed by SPSS 16.0 (SPSS Inc, Chicago, IL, USA). Unpaired $t$ test and one-way ANOVA were utilized to detect the statistical significance. Generally, the differences between the means of study data were statistically significant if $P<0.05$.

\section{ACKNOWLEDGEMENTS}

This study was conducted at State Key Laboratory of Oral Diseases, West China School of Stomatology, Sichuan University and IVC Experimental Animal Center of Public Health, Sichuan University. This work was supported by grants from the National Natural Science Foundation of China (grant Nos. 81771068, 81670980, and
81800964), and Sichuan International Science and Technology Innovation Cooperation (Grant No. 2020YFH0010).

\section{AUTHOR CONTRIBUTIONS}

Tao Hu and Lei Lei designed the experiments, analyzed the data, and wrote this paper; Yalan Deng and Yingming Yang performed the experiments, analyzed the data, and wrote this paper; Bin Zhang, Hong Chen, Yangyu Lu and Shirui Ren contributed to data acquisition and analysis. All authors gave final approval and agreed to be accountable for all aspects of the work.

\section{ADDITIONAL INFORMATION}

Supplementary information The online version contains supplementary material available at https://doi.org/10.1038/s41368-021-00149-x.

Competing interests: The authors declare no competing interests.

Statement of ethics: Animal study in this paper conformed to the ethical guidelines of the IVC Experimental Animal Center of Public Health, Sichuan University (Chengdu, China), and was approved by the Ethics Committee of West China Hospital of Stomatology, Sichuan University (NO: WCHSIRB-D-2019-144).

\section{REFERENCES}

1. Bowen, W. H., Burne, R. A., Wu, H. \& Koo, H. Oral biofilms: pathogens, matrix, and polymicrobial interactions in microenvironments. Trends Microbiol. 26, 229-242 (2018). 
2. Yin, W. et al. Oral health status in Sichuan Province: findings from the oral health survey of Sichuan, 2015-2016. Int. J. Oral. Sci. 9, 10-15 (2017).

3. Peres, M. A. et al. Oral diseases: a global public health challenge. Lancet $\mathbf{3 9 4}$ 249-260 (2019).

4. Zhou, Y. et al. Evaluating Streptococcus mutans strain dependent characteristics in a polymicrobial biofilm community. Front. Microbiol. 9, 1498 (2018).

5. Xiao, J., Fiscella, K. A. \& Gill, S. R. Oral microbiome: possible harbinger for children's health. Int. J. Oral. Sci. 12, 12 (2020).

6. Hobley, L., Harkins, C., MacPhee, C. E. \& Stanley-Wall, N. R. Giving structure to the biofilm matrix: an overview of individual strategies and emerging common themes. FEMS Microbiol. Rev. 39, 649-669 (2015).

7. Flemming, H. C. et al. Biofilms: an emergent form of bacterial life. Nat. Rev. Microbiol. 14, 563-575 (2016).

8. Cugini, C., Shanmugam, M., Landge, N. \& Ramasubbu, N. The role of exopolysaccharides in oral biofilms. J. Dent. Res. 98, 739-745 (2019).

9. Liu, Y., Ren, Z., Hwang, G. \& Koo, H. Therapeutic strategies targeting cariogenic biofilm microenvironment. Adv. Dent. Res. 29, 86-92 (2018).

10. Jiao, Y., Tay, F. R., Niu, L. N. \& Chen, J. H. Advancing antimicrobial strategies for managing oral biofilm infections. Int J. Oral. Sci. 11, 28 (2019).

11. Garcia, S. S. et al. Targeting of Streptococcus mutans biofilms by a novel small molecule prevents dental caries and preserves the oral microbiome. J. Dent. Res. 96, 807-814 (2017)

12. Naha, P. C. et al. Dextran-coated iron oxide nanoparticles as biomimetic catalysts for localized and pH-activated biofilm disruption. ACS Nano 13, 4960-4971 (2019).

13. Simon-Soro, A. \& Mira, A. Solving the etiology of dental caries. Trends Microbiol. 23, 76-82 (2015).

14. Mao, M. Y. et al. The rnc gene promotes exopolysaccharide synthesis and represses the vicRKX gene expressions via microRNA-size small RNAs in Streptococcus mutans. Front. Microbiol. 7, 687 (2016).

15. Wagner, C. et al. Genetic analysis and functional characterization of the Streptococcus pneumoniae vic operon. Infect. Immun. 70, 6121-6128 (2002).

16. Lei, L. et al. Modulation of biofilm exopolysaccharides by the Streptococcus mutans vicX gene. Front. Microbiol. 6, 1432 (2015).

17. Lei, L. et al. Activity of Streptococcus mutans VicR is modulated by antisense RNA. J. Dent. Res. https://doi.org/10.1177/0022034518781765 (2018).

18. Lei, L. et al. Carbohydrate metabolism regulated by antisense vicR RNA in cariogenicity. J. Dent. Res. 99, 204-213 (2020).

19. Zschiedrich, C. P., Keidel, V. \& Szurmant, H. Molecular mechanisms of twocomponent signal transduction. J. Mol. Biol. 428, 3752-3775 (2016).

20. Deng, D. M., Liu, M. J., ten Cate, J. M. \& Crielaard, W. The VicRK system of Streptococcus mutans responds to oxidative stress. J. Dent. Res. 86, 606-610 (2007).

21. Senadheera, D. B. et al. Regulation of bacteriocin production and cell death by the VicRK signaling system in Streptococcus mutans. J. Bacteriol. 194, 1307-1316 (2012).

22. Ayala, E. et al. A biochemical characterization of the DNA binding activity of the response regulator VicR from Streptococcus mutans. PLOS ONE 9, e108027 (2014).

23. Senadheera, M. D. et al. A VicRK signal transduction system in Streptococcus mutans affects gtfBCD, gbpB, and ftf expression, biofilm formation, and genetic competence development. J. Bacteriol. 187, 4064-4076 (2005).

24. Alves, L. A. et al. The two-component system VicRK regulates functions associated with Streptococcus mutans resistance to complement immunity. Mol. Oral. Microbiol. 32, 419-431 (2017)

25. Zhuang, P. L. et al. Relationship between the genetic polymorphisms of vicR and vick Streptococcus mutans genes and early childhood caries in two-year-old children. BMC Oral Health 18, 39 (2018).

26. Wang, $C$. et al. Mechanistic insights revealed by the crystal structure of a histidine kinase with signal transducer and sensor domains. PLoS Biol. 11, e1001493 (2013).

27. Horev, B. et al. pH-activated nanoparticles for controlled topical delivery of farnesol to disrupt oral biofilm virulence. ACS Nano 9, 2390-2404 (2015).

28. Flemming, H. C. \& Wingender, J. The biofilm matrix. Nat. Rev. Microbiol. 8, 623-633 (2010).

29. Davies, D. Understanding biofilm resistance to antibacterial agents. Nat. Rev. Drug Discov. 2, 114-122 (2003)

30. Duque, C. et al. Downregulation of $\mathrm{GbpB}$, a component of the VicRK regulon, affects biofilm formation and cell surface characteristics of Streptococcus mutans. Infect. Immun. 79, 786-796 (2011).

31. Donlan, R. M. \& Costerton, J. W. Biofilms: survival mechanisms of clinically relevant microorganisms. Clin. Microbiol. Rev. 15, 167-193 (2002).

32. Schilcher, K. \& Horswill, A. R. Staphylococcal biofilm development: structure, regulation, and treatment strategies. Microbiol. Mol. Biol. Rev. 84, e00026-19 (2020).

33. Senadheera, D. et al. Inactivation of VicK affects acid production and acid survival of Streptococcus mutans. J. Bacteriol. 191, 6415-6424 (2009).
34. Karygianni, L., Ren, Z., Koo, H. \& Thurnheer, T. Biofilm matrixome: extracellular components in structured microbial communities. Trends Microbiol. 28, 668-681 (2020).

35. Marsh, P. D. \& Zaura, E. Dental biofilm: ecological interactions in health and disease. J. Clin. Periodontol. 44, S12-s22 (2017).

36. Hwang, G. et al. Candida albicans mannans mediate Streptococcus mutans exoenzyme GtfB binding to modulate cross-kingdom biofilm development in vivo. PLoS Pathog. 13, e1006407 (2017).

37. Hwang, G. et al. Simultaneous spatiotemporal mapping of in situ $\mathrm{pH}$ and bacterial activity within an intact 3D microcolony structure. Sci. Rep. 6, 32841 (2016).

38. Gao, Y. et al. Size and charge adaptive clustered nanoparticles targeting the biofilm microenvironment for chronic lung infection management. ACS Nano 14, 5686-5699 (2020).

39. Sims, K. R. Jr et al. Dual antibacterial drug-loaded nanoparticles synergistically improve treatment of Streptococcus mutans biofilms. Acta Biomater. 115 418-431 (2020).

40. Wang, Y. et al. Oral biofilm elimination by combining iron-based nanozymes and hydrogen peroxide-producing bacteria. Biomater. Sci. 8, 2447-2458 (2020).

41. Fulaz, S., Vitale, S., Quinn, L. \& Casey, E. Nanoparticle-biofilm interactions: the role of the EPS matrix. Trends Microbiol. 27, 915-926 (2019).

42. Qi, J. \& Kim, S. M. Effects of the molecular weight and protein and sulfate content of Chlorella ellipsoidea polysaccharides on their immunomodulatory activity. Int. J. Biol. Macromol. 107, 70-77 (2018)

43. Hou, N. et al. Polysaccharides and their depolymerized fragments from Costaria costata: molecular weight and sulfation-dependent anticoagulant and FGF/FGFR signal activating activities. Int. J. Biol. Macromol. 105, 1511-1518 (2017).

44. Sun, L., Wang, C., Shi, Q. \& Ma, C. Preparation of different molecular weight polysaccharides from Porphyridium cruentum and their antioxidant activities. Int. J. Biol. Macromol. 45, 42-47 (2009).

45. Liu, W. et al. Characterization and antioxidant activity of two low-molecularweight polysaccharides purified from the fruiting bodies of Ganoderma lucidum. Int. J. Biol. Macromol. 46, 451-457 (2010).

46. Xu, X. et al. Gastric protective activities of sea cucumber fucoidans with different molecular weight and chain conformations: a structure-activity relationship investigation. J. Agric. Food Chem. 66, 8615-8622 (2018).

47. Zeng, L. \& Burne, R. A. Comprehensive mutational analysis of sucrosemetabolizing pathways in Streptococcus mutans reveals novel roles for the sucrose phosphotransferase system permease. J. Bacteriol. 195, 833-843 (2013).

48. Castillo Pedraza, M. C. et al. Modulation of lipoteichoic acids and exopolysaccharides prevents streptococcus mutans biofilm accumulation. Molecules. 25 2232 (2020).

49. Kan, L., Chai, Y., Li, X. \& Zhao, M. Structural analysis and potential anti-tumor activity of Sporisorium reilianum (Fries) polysaccharide. Int. J. Biol. Macromol. 153, 986-994 (2020).

50. Xu, Z. et al. Characteristics and bioactivities of different molecular weight polysaccharides from camellia seed cake. Int. J. Biol. Macromol. 91, 1025-1032 (2016).

51. Chen, Q. L. et al. Laser microdissection hyphenated with high performance gel permeation chromatography-charged aerosol detector and ultra performance liquid chromatography-triple quadrupole mass spectrometry for histochemical analysis of polysaccharides in herbal medicine: Ginseng, a case study. Int. J. Biol. Macromol. 107, 332-342 (2018).

52. Duan, G. L. \& Yu, X. B. Isolation, purification, characterization, and antioxidant activity of low-molecular-weight polysaccharides from Sparassis latifolia. Int. J. Biol. Macromol. 137, 1112-1120 (2019).

53. Paula, A. J., Hwang, G. \& Koo, H. Dynamics of bacterial population growth in biofilms resemble spatial and structural aspects of urbanization. Nat. Commun. 11, 1354 (2020).

54. Lemos, J. A. et al. The biology of Streptococcus mutans. Microbiol. Spectr. 7, https://doi.org/10.1128/microbiolspec.GPP3-0051-2018 (2019).

55. Florez Salamanca, E. J. \& Klein, M. I. Extracellular matrix influence in Streptococcus mutans gene expression in a cariogenic biofilm. Mol. Oral. Microbiol. 33, 181-193 (2018).

56. Gushchin, I. et al. Mechanism of transmembrane signaling by sensor histidine kinases. Science. 356, eaah6345 (2017)

57. Senadheera, M. D. et al. The Streptococcus mutans vicX gene product modulates $\mathrm{gtfB} / \mathrm{C}$ expression, biofilm formation, genetic competence, and oxidative stress tolerance. J. Bacteriol. 189, 1451-1458 (2007).

58. Biswas, I. \& Mohapatra, S. S. CovR alleviates transcriptional silencing by a nucleoid-associated histone-like protein in Streptococcus mutans. J. Bacteriol. 194, 2050-2061 (2012).

59. Alves, L. A. et al. CovR and VicRKX regulate transcription of the collagen binding protein Cnm of Streptococcus mutans. J. Bacteriol. 200, e00141-18 (2018).

60. Negrini, T. C. et al. Influence of VicRK and CovR on the interactions of Streptococcus mutans with phagocytes. Oral Dis. 18, 485-493 (2012). 
61. Biswas, S. \& Biswas, I. Regulation of the glucosyltransferase (gtfBC) operon by CovR in Streptococcus mutans. J. Bacteriol. 188, 988-998 (2006).

62. Idone, V. et al. Effect of an orphan response regulator on Streptococcus mutans sucrose-dependent adherence and cariogenesis. Infect. Immun. 71, 4351-4360 (2003).

63. Lee, S. F., Delaney, G. D. \& Elkhateeb, M. A two-component covRS regulatory system regulates expression of fructosyltransferase and a novel extracellular carbohydrate in Streptococcus mutans. Infect. Immun. 72, 3968-3973 (2004).

64. Chong, P., Drake, L. \& Biswas, I. Modulation of covR expression in Streptococcus mutans UA159. J. Bacteriol. 190, 4478-4488 (2008).

65. Downey, J. S. et al. In vitro manganese-dependent cross-talk between Streptococcus mutans VicK and GcrR: implications for overlapping stress response pathways. PLOS ONE 9, e115975 (2014).

66. Wang, S. et al. Dissecting the role of Vick phosphatase in aggregation and biofilm formation of Streptococcus mutans. J. Dent. Res. 100, 631-638 (2021).

67. Burne, R. A. et al. Cariogenicity of Streptococcus mutans strains with defects in fructan metabolism assessed in a program-fed specific-pathogen-free rat model. J. Dent. Res. 75, 1572-1577 (1996).

68. Alves, L. A. et al. CovR regulates Streptococcus mutans susceptibility to complement immunity and survival in blood. Infect. Immun. 84, 3206-3219 (2016).

69. Koo, H., Falsetta, M. L. \& Klein, M. I. The exopolysaccharide matrix: a virulence determinant of cariogenic biofilm. J. Dent. Res. 92, 1065-1073 (2013).

70. Stipp, R. N. et al. CovR and VicRK regulate cell surface biogenesis genes required for biofilm formation in Streptococcus mutans. PLOS ONE 8, e58271 (2013).

71. Zhao, W. et al. Effect of sucrose concentration on sucrose-dependent adhesion and glucosyltransferase expression of S. mutans in children with severe earlychildhood caries (S-ECC). Nutrients 6, 3572-3586 (2014).

72. Decker, E. M., Klein, C., Schwindt, D. \& von Ohle, C. Metabolic activity of Streptococcus mutans biofilms and gene expression during exposure to xylitol and sucrose. Int J. Oral. Sci. 6, 195-204 (2014).

73. Hanada, N. \& Kuramitsu, H. K. Isolation and characterization of the Streptococcus mutans gtfD gene, coding for primer-dependent soluble glucan synthesis. Infect. Immun. 57, 2079-2085 (1989).

74. Klein, M. I. et al. Streptococcus mutans-derived extracellular matrix in cariogenic oral biofilms. Front. Cell Infect. Microbiol. 5, 10 (2015).

75. Zhu, F., Zhang, H. \& Wu, H. Glycosyltransferase-mediated sweet modification in oral streptococci. J. Dent. Res. 94, 659-665 (2015).

76. Ooshima, T. et al. Contributions of three glycosyltransferases to sucrose-dependent adherence of Streptococcus mutans. J. Dent. Res. 80, 1672-1677 (2001).

77. Stipp, R. N. et al. Transcriptional analysis of gtfB, gtfC, and $\mathrm{gbpB}$ and their putative response regulators in several isolates of Streptococcus mutans. Oral. Microbiol. Immunol. 23, 466-473 (2008).

78. Abranches, J. et al. Biology of oral streptococci. Microbiol. Spectr. 6, https://doi. org/10.1128/microbiolspec.GPP3-0042-2018 (2018).

79. McNeill, K. \& Hamilton, I. R. Acid tolerance response of biofilm cells of Streptococcus mutans. FEMS Microbiol. Lett. 221, 25-30 (2003).

80. Lau, P. C. et al. PCR ligation mutagenesis in transformable streptococci: application and efficiency. J. Microbiol. Methods 49, 193-205 (2002).
81. Weidmann, S. et al. Production of the small heat shock protein Lo18 from Oenococcus oeni in Lactococcus lactis improves its stress tolerance. Int. J. Food Microbiol. 247, 18-23 (2017).

82. Fong, S. A. et al. Activity of bacteriophages in removing biofilms of Pseudomonas aeruginosa isolates from chronic rhinosinusitis patients. Front. Cell Infect. Microbiol. 7, 418 (2017).

83. Marka, S. \& Anand, S. Feed substrates influence biofilm formation on reverse osmosis membranes and their cleaning efficiency. J. Dairy Sci. 101, 84-95 (2018).

84. Kannan, A. et al. Nanoscale investigation on Pseudomonas aeruginosa biofilm formed on porous silicon using atomic force microscopy. Scanning 36, 551-553 (2014).

85. Neu, T. R. \& Lawrence, J. R. Innovative techniques, sensors, and approaches for imaging biofilms at different scales. Trends Microbiol. 23, 233-242 (2015).

86. da Silva, B. R. et al. Antibacterial activity of a novel antimicrobial peptide [W7] KR12-KAEK derived from KR-12 against Streptococcus mutans planktonic cells and biofilms. Biofouling 33, 835-846 (2017).

87. Zhang, K. et al. Effect of antibacterial dental adhesive on multispecies biofilms formation. J. Dent. Res. 94, 622-629 (2015).

88. Mao, M. Y. et al. The regulator gene rnc is closely involved in biofilm formation in Streptococcus mutans. Caries Res. 52, 347-358 (2018).

89. Blanco, Y. et al. Environmental parameters, and not phylogeny, determine the composition of extracellular polymeric substances in microbial mats from extreme environments. Sci. Total Environ. 650, 384-393 (2019).

90. Bowen, W. H. Rodent model in caries research. Odontology 101, 9-14 (2013).

91. Yucesoy, D. T. et al. Early caries in an in vivo model: structural and nanomechanical characterization. J. Dent. Res. https://doi.org/10.1177/0022034518789898 (2018).

92. Keyes, P. H. Dental caries in the molar teeth of rats. I. Distribution of lesions induced by high-carbohydrate low-fat diets. J. Dent. Res. 37, 1077-1087 (1958).

93. Monette, P. et al. Autoregulation of the S. mutans SloR metalloregulator is constitutive and driven by an independent promoter. J. Bacteriol. 200, e00214-18 (2018).

94. Bustin, S. A. et al. The MIQE guidelines: minimum information for publication of quantitative real-time PCR experiments. Clin. Chem. 55, 611-622 (2009).

(i) Open Access This article is licensed under a Creative Commons Attribution 4.0 International License, which permits use, sharing, adaptation, distribution and reproduction in any medium or format, as long as you give appropriate credit to the original author(s) and the source, provide a link to the Creative Commons license, and indicate if changes were made. The images or other third party material in this article are included in the article's Creative Commons license, unless indicated otherwise in a credit line to the material. If material is not included in the article's Creative Commons license and your intended use is not permitted by statutory regulation or exceeds the permitted use, you will need to obtain permission directly from the copyright holder. To view a copy of this license, visit http://creativecommons. org/licenses/by/4.0/.

(c) The Author(s) 2021 